\title{
Margetuximab for the treatment of HER2-positive metastatic breast cancer
}

\author{
Paolo Tarantino ${ }^{a, b}$, Stefania Morganti ${ }^{\mathrm{a}, \mathrm{b}}$, Jacopo Uliano ${ }^{\mathrm{a}, \mathrm{b}}$, Federica Giugliano ${ }^{\mathrm{a}, \mathrm{b}}$, Edoardo Crimini ${ }^{\mathrm{a}, \mathrm{b}}$ \\ and Giuseppe Curigliano ${ }^{\mathrm{a}, \mathrm{b}}$

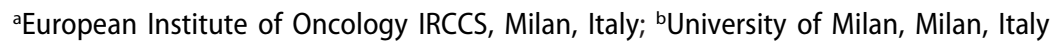

ABSTRACT

Introduction: No specific standard treatment is currently recommended for HER2-positive advanced breast cancer (BC) patients progressing to dual HER2 blockade and to trastuzumab emtansine (TDM-1). However, several novel anti-HER2 agents are emerging and rapidly revolutionizing this setting. Among these, the FC-engineered monoclonal antibody margetuximab has recently demonstrated to slightly improve progression-free survival (PFS) compared with trastuzumab, when combined with chemotherapy for pretreated HER2-positive advanced BC.

Areas covered: The present review article recapitulates the clinical development of margetuximab, critically discussing its implications in the current landscape of BC treatment algorithms.

Expert opinion: The clinical role of Margetuximab can only be interpreted in view of the rapidly evolving treatment landscape for pretreated HER2-positive advanced BC. Indeed, the recently approved anti-HER2 agents tucatinib and trastuzumab deruxtecan currently represent appealing options for the post-TDM1 setting, while margetuximab may have a role after progression to the abovementioned agents, in case of a future approval. Regardless of its clinical uptake, it should be noted that the development of margetuximab has relevantly improved our biological understanding of HER2-positive BC, highlighting the implication of patient's genotype in determining treatment outcomes, as well as the relevance of antibodydependent cellular cytotoxicity (ADCC) in the context of HER2-blockade.
ARTICLE HISTORY

Received 2 October 2020

Accepted 24 November 2020

\section{KEYWORDS}

Margetuximab; mgah22; her2; breast cancer; sophia trial

\section{Introduction}

About $15-20 \%$ of breast cancers (BC) overexpress HER2 as consequence of ERBB2 gene amplification[1]. The discovery and clinical implementation of a wide variety of agents targeting the HER2 pathway has radically changed the prognosis of this subgroup of patients in the last decades, both in the early and advanced setting $[2,3]$. In particular, dual HER2 blockade with trastuzumab and pertuzumab combined with chemotherapy is currently considered the preferred first-line treatment for advanced HER2-positive BC, whereas the antibody-drug conjugate (ADC) trastuzumab-emtansine (TDM1) is currently considered a standard second-line choice[4]. Although no standard third-line treatment is recommended by current guidelines, multiple novel anti-HER2 compounds have recently emerged, rapidly revolutionizing this treatment setting. Indeed, the novel antiHER2 ADC trastuzumab deruxtecan [5] and the anti-HER2 tyrosine kinase inhibitors (TKIs) tucatinib [6] and neratinib [7] were recently approved by FDA, enlarging the treatment arsenal of pretreated HER2-positive BC patients[4]. Among these emerging agents, the anti-HER2 monoclonal antibody (mAb) margetuximab has reached the advanced stage of development, thanks to the promising results shown in several early phase trials.

The purpose of this review is to recapitulate the clinical development of margetuximab, and to critically discuss its implications in the current landscape of BC treatment algorithms.

\subsection{Current landscape in TDM-1 pretreated patients}

As previously mentioned, a standard treatment strategy for $\mathrm{BC}$ patients pretreated with trastuzumab, pertuzumab and TDM-1 is yet to be determined. Several traditional options are suggested by current guidelines, including chemotherapy combined with trastuzumab, with lapatinib, or a chemo-free treatment with the two anti-HER2 agents[8]. More recently, a number of approvals have revolutionized the field, providing novel valuable options in this setting (Table 1). Indeed, the combination of tucatinib, trastuzumab and capecitabine was approved by the Food \& Drugs Administration (FDA) for pretreated HER2-positive advanced BC patients, based on the overall survival (OS) benefit demonstrated in the phase III HER2CLIMB trial[6]. Tucatinib activity was particularly evident in the population of BC patients with brain metastasis, including those not previously treated with radiotherapy[9]. A second TKI was approved by FDA in the same setting, namely neratinib combined with capecitabine, although the regimen did not show to improve survival compared with lapatinib and capecitabine[7]. Finally, the novel ADC trastuzumab deruxtecan was granted an accelerated approval by FDA for TDM-1 pretreated patients, based on the impressive objective response rate (ORR) and progression-free survival (PFS) observed in the single-arm DESTINY-Breast01 trial[5]. The phase 3 DESTINY-Breast02 trial is currently ongoing, to confirm the role of trastuzumab deruxtecan after progression to TDM-1 (ClinicalTrials.gov ID: NCT03523585). 
With a rapidly enlarging arsenal of anti-HER2 regimens for pretreated patients, guidelines are currently undergoing a wide revision, and may in the future lead to an improved tailoring of treatments based on patients' characteristics and preferences. Furthermore, some of the abovementioned novel drugs are being tested in second-line against TDM-1 monotherapy (e.g. DESTINY-Breast03 phase 3 trial, HER2CLIMB-02 phase 3 trial), and ongoing immuno-oncology trials may also redefine current treatment algorithms in the future[10]. It should be noted, however, that none of the abovementioned novel drugs is yet approved by the European Medicine Agency (EMA).

\subsection{Margetuximab: pharmacodynamics and pharmacokinetics}

Margetuximab (also known as MGAH22) is a human/mouse chimeric lgG1 monoclonal antibody targeting HER2 receptor. Based on the murine precursor of trastuzumab, it has similar epitope binding proprieties, but with an engineered Fc domain: the substitution of five aminoacid into the IgG1 Fc domain improves binding to FcyRIIIA (CD16A), a low-affinity stimulatory receptor found on macrophages and natural killer cells, and reduces binding to the inhibitory Fc receptor FcYRIIB (CD32B) (Figure 1) [11] No difference in binding to the high-affinity activating FcyRI (CD64) was instead observed in preclinical studies[12].

More in detail, CD16A is encoded by two alleles differing in the codon for amino acid 158: a higher-affinity valine (V) variant and a lower-affinity phenylalanine $(F)$ variant[13]. The increase in affinity to both the allotypes of CD16A ultimately leads to a greater antibody-dependent cellular cytotoxicity (ADCC) activation, namely the killing of targeted cells by activated effector cells (NK lymphocytes, macrophages, neutrophils), which has been previously demonstrated to be a relevant player in the context of targeting HER2 [14,15]. Preclinical studies demonstrate that the optimized Fc domain confers enhanced ADCC against all HER2+ tumor cell tested compared with trastuzumab, including in trastuzumab resistant and HER2 low expressing cells, defined as those with an HER" immunohistochemical score of 1 + or $2+$ with a negative in-situ hybridization test $[12,16]$. Of note, the greatest improvement in ADCC activation occurred in presence of the CD16A-158 F, the low-binding allele[12].

The phase 1 study [17] elucidates Margetuximab's pharmacokinetics: a bi-compartment model and Michaelis - Menten elimination, with a terminal half-live estimated at 15.5 days (about half of that observed with trastuzumab [18]). The recommended dose, used for the subsequent studies, was $15 \mathrm{mg} / \mathrm{kg}$ Q3W or $6.0 \mathrm{mg} / \mathrm{kg} \mathrm{QW}$.

\subsection{Early clinical development}

The clinical development of Margetuximab started on 2010, when the first-in-human phase I trial was launched, sponsored by MacroGenics. The trial enrolled 66 patients affected by HER2positive advanced solid tumors for whom no standard therapy was available, including mostly breast (27 patients) and gastroesophageal (20 patients) adenocarcinomas. Interestingly, HER2 positivity was assessed only by immunohistochemistry, and patients scored $3+$ and $2+$ could be included, regardless of HER2 amplification status. Margetuximab demonstrated to be well tolerated, as the most frequent toxicities were grade 1 and grade 2, including especially pyrexia, nausea, anemia, diarrhea, and fatigue. The compound also showed antitumor activity, as $64 \%$ of the 60 patients who were evaluable for tumor response experienced a stability of disease $(52 \%)$ or a partial response (12\%). Noticeably, all the patients who obtained a response had HER2 expression assessed as 3+ at immunohistochemistry or harbored amplification at FISH test. Median PFS was 3.5 months among all the included patients and 4.5 months among BC patients[17].

Following the phase I study, several further early phase studies were initiated in various diseases.

A phase II trial (ClinicalTrials.gov ID NCT01828021) was designed and started enrollment in 2013, in order to explore the efficacy of Margetuximab in HER2-low BC patients, defined as those showing $2+$ by immunohistochemistry without amplification at FISH test. A total of 25 patients affected by advanced BC and who received at least two lines of therapy were included, with efficacy results available for 22 patients. No responses were achieved in these patients, with only six disease stabilizations observed in the trial[19]. Furthermore, as a confirmation of the worldwide rising awareness of potentialities of this novel antiHER2 compound, a phase I (NCT04398108) and a phase II (NCT04262804) studies are currently investigating safety and efficacy of Margetuximab in Chinese HER2-positive BC patients.

The compound was also explored in combination with immune checkpoint inhibitors (ICls): a phase lb/ll study of margetuximab combined with pembrolizumab for gastric and gastroesophageal cancers with HER2 overexpression (HER2 3+ or amplification at FISH test) enrolled 95 patients pretreated with Trastuzumab. The results showed that the combination of antiPD1 and anti-HER2 therapy is acceptably safe and discretely tolerated; the most frequent grade 3 or 4 adverse events related to the treatment were anemia (4\%) and infusion-related reactions (3\%), followed by autoimmune hepatitis (2\%). Concerning efficacy analysis, median PFS was 2.7 months, with a median OS of 12 months; ORR was $18 \%$, with a disease control rate of $53 \%$. It is necessary to emphasize that PFS and OS were significantly longer for patients with HER2 $3+$ at immunohistochemistry. These results warranted further studies, taking into account the biological plausibility of synergic action of adaptive and natural immunity activation, respectively, due to PD1 blockade with 


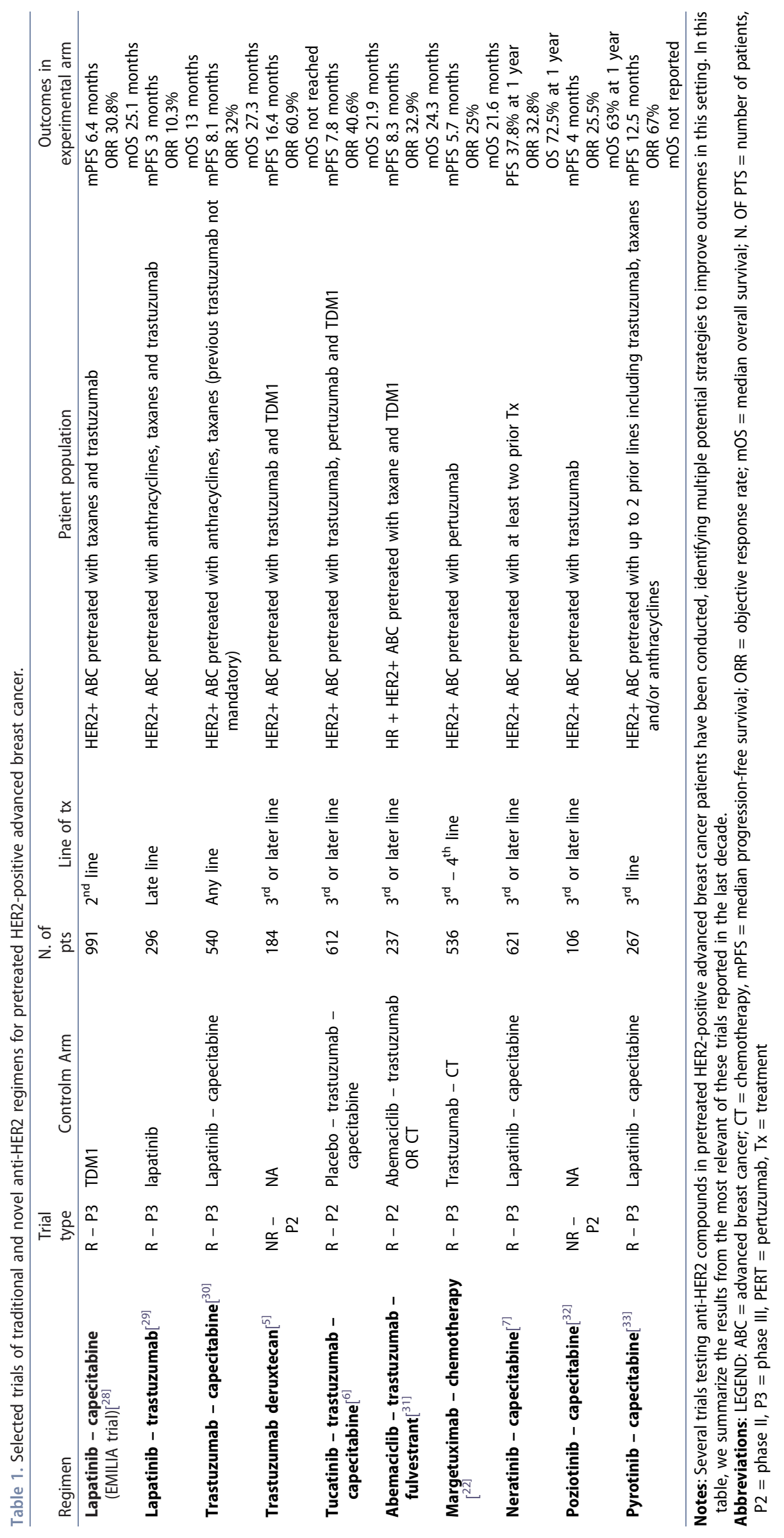




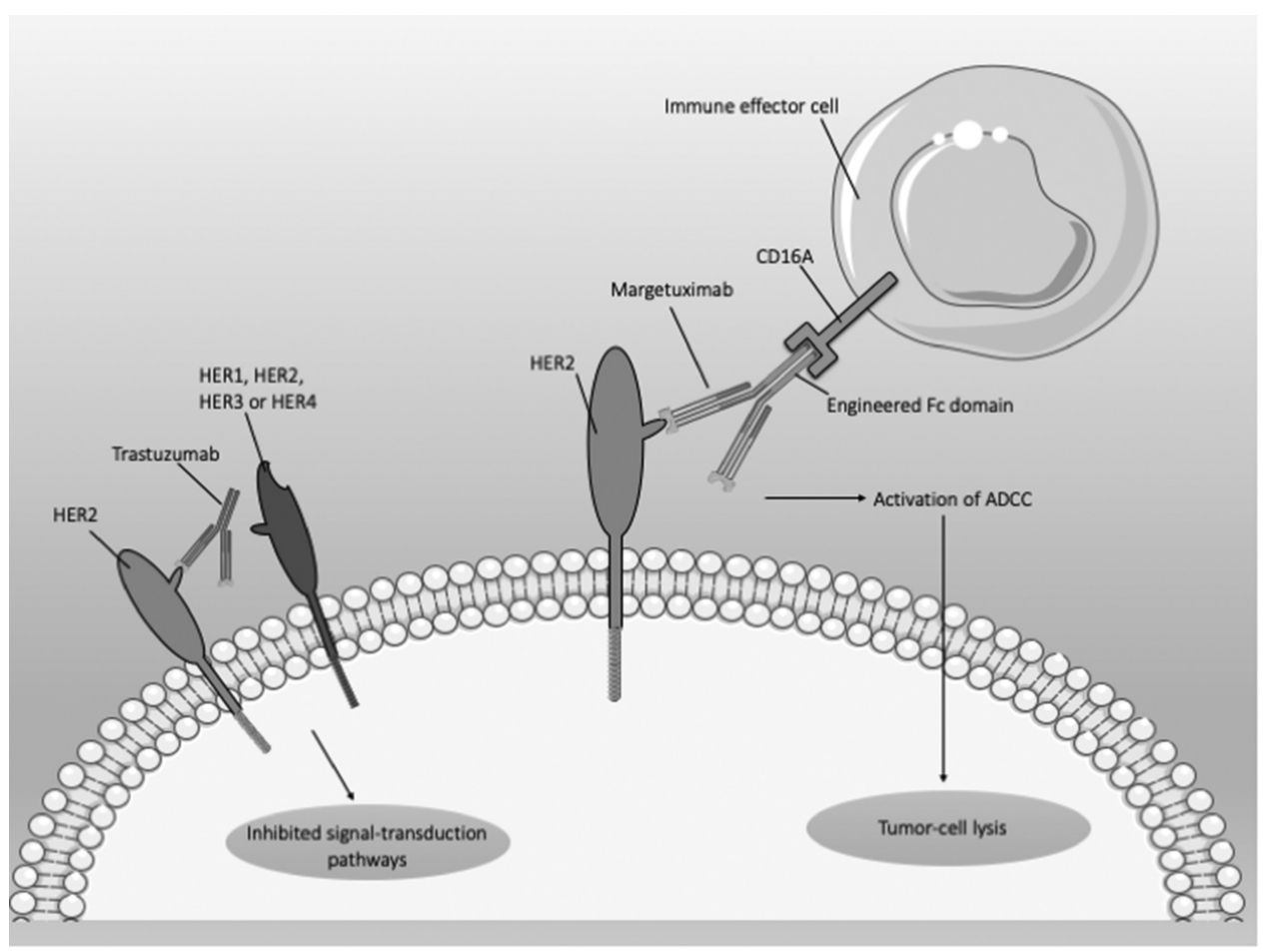

Figure 1. Mechanism of action of Margetuximab. On the left, trastuzumab inhibits homodimerization or heterodimerization with another member of HER family, thus reducing HER2 signaling pathway which leads to cell proliferation, survival, motility and invasiveness. Margetuximab has similar epitope binding proprieties, with an engineered Fc domain which improves binding to both the the allotypes of activating Fc receptor FcyllIA (CD16A) and reduces binding to the inhibitory Fc receptor FcYRIIB (CD32B), leading to a greater ADCC activation and a subsequent superior cytotoxicity compared with trastuzumab. ADCC: antibody-dependent cellmediated cytotoxicity; HER: human epidermal growth factor receptor. This figure was created using Servier Medical Art templates, which are licensed under a Creative Commons Attribution 3.0 Unported License;.https://smart.servier.com

pembrolizumab and ADCC eliciting via CD16a activation by margetuximab[20]. In this perspective, the MAHOGANY phase II/III trial is exploring the safety and efficacy of the combination of margetuximab and $\mathrm{ICls}$ in the first-line setting for patients affected by HER2-positive gastro-esophageal adenocarcinoma, disclosing a possible chemo-free scenario for these patients.

Finally, the encouraging results achieved by the compound in advanced $B C$ patients prompted the initiation of studies in the early setting. In particular, a newly-designed Phase II study (ClinicalTrials.gov ID: NCT04425018-MARGOT) is currently comparing the activity of margetuximab plus pertuzumab and paclitaxel to the standard of care treatment of trastuzumab, pertuzumab, and paclitaxel in the neoadjuvant setting for HER2-positive BC patients. The primary endpoint is pathological complete response and the estimated primary completion date is 2024.

On the whole, the attempt to expand margetuximab indications is actually supported by increasing data on its activity and safety in various cancers, as showed by the numerous completed and ongoing clinical trials.

\subsection{Late clinical development: the SOPHIA trial}

Based on the promising efficacy demonstrated by the antibody in early phase trials enrolling BC patients, a confirmatory phase III trial was initiated in 2015.
SOPHIA was a randomized, open-label phase III trial testing the combination of margetuximab plus chemotherapy versus trastuzumab plus chemotherapy in patients with HER2-positive metastatic BC. All patients were pretreated with at least 2 antiHER2 regimens, including pertuzumab, and 1-3 lines of therapy of systemic therapy for HER2+ metastatic BC[21]. Patients enrolled were randomized 1:1 to receive margetuximab or trastuzumab along with capecitabine, eribulin, gemcitabine, or vinorelbine, as per investigator choice. Stratification factors included number of metastatic sites $(\leq 2,>2)$, previous lines of treatment for metastatic $B C(\leq 2,>2)$, and administered chemotherapy. Primary endpoints were PFS and OS.

536 patients were included in the intention-to-treat (ITT) population. Most patients received vinorelbine as combination chemotherapy (36\%), followed by capecitabine (27\%), eribulin $(25 \%)$ and gemcitabine (12\%). All patients received both trastuzumab and pertuzumab as previous anti-HER2 agents, along with T-DM1 in more than $90 \%$ of cases in both arms. Two-third of patients received $\leq 2$ lines of therapy for metastatic disease. $62 \%$ had HR+/HER2- BC[22].

The first PFS analysis has been published in 2019 (data cutoff 10 October 2018). After 265 PFS events, margetuximab showed to significantly prolong PFS over trastuzumab (median 5.8 vs 4.9 months, HR $0.76 ; 95 \% \mathrm{Cl} 0.59-0.98 ; \mathrm{p} 0.033$ ). A benefit favoring margetuximab over trastuzumab was observed in all chemotherapy arms, and more pronounced 
for patients receiving eribulin ( $\mathrm{HR} 0.66 ; \mathrm{Cl} 0.42-1.05)$ and gembitabine (HR 0.58; $\mathrm{Cl} 0.29-1.18$ ), even if statistical significance was not reached in any subgroup[21].

Observed antitumor responses were also higher in patients in the experimental arm (ORR $22 \%$ vs $16 \%$ ). Interestingly, benefit was more pronounced in patients with a CD16A genotype containing a $158 \mathrm{~F}$ allele (median PFS 6.9 vs 5.1 months, HR 0.68; 95\% Cl 0.52-0.90; p 0.005). [21]

A second interim analysis conducted after 270 deaths (70\%) confirmed the benefit in terms of both PFS (HR 0.71, p 0.0006) and ORR ( 25.2 vs $13.7 \%$, p 0.0006). However, no statistically significant advantage was observed in terms of OS, with an absolute increase of 1.8 months for the margetuximab versus trastuzumab (median OS 21.6 vs 19.8, HR 0.89; p 0.326). An increased benefit was observed for CD16A-185 F carriers in pre-specified exploratory analysis, even if still not significant (mOS 23.7 vs 19.4, HR 0.79, p 0.087). Of note, CD16A-185 F carriers represented the wide majority of the enrolled population, accounting for about $85 \%$ of the patients[22].

Overall, the combination of margetuximab plus chemotherapy was relatively well tolerated, with toxicities comparable to what observed in the standard arm in all chemotherapy groups. Grade $\geq 3$ AEs occurred in $53.8 \%$ and $52.6 \%$ of patients receiving margetuximab and trastuzumab, respectively. Serious AEs were recorded in $16.3 \%$ and $18.4 \%$ of patients. More patients on margetuximab than trastuzumab discontinued chemotherapy because of toxicity ( $11 \%$ vs $6.4 \%)$, while only 3 of them were considered probably or definitely related to antibody therapy (seroma and infusion-related reaction for margetuximab, pneumonia for trastuzumab) $[22,23]$.

Extended publication of the SOPHIA trial is awaited.

\subsection{Regulatory aspects}

Although a biologics license application (BLA) for margetuximab was submitted to the FDA in December 2019, the compound has not yet been granted any approval for the treatment of $B C$, neither for other cancer types. Based on the outcomes of the SOPHIA trial, and based on FDA approval history in the same setting, a future approval for margetuximab for pretreated HER2-positive advanced BC patients may be hypothesized. Indeed, the TKI neratinib was approved in the same setting on the basis of a relatively small PFS benefit demonstrated in the NALA trial, without showing any statistically significant OS improvement[7]. Less clear is if the CD16A genotype analysis will impact regulatory decisions, when a PFS benefit could be demonstrated in the intent-to-treat population of the SOPHIA trial.

No application was instead submitted to date to the European Medicine Agency to our knowledge.

\section{Expert Opinion}

The impact of the SOPHIA trial on current treatment algorithms can only be interpreted in view of the rapidly evolving treatment landscape for pretreated HER2-positive advanced BC[24]. Indeed, although margetuximab could demonstrate a small PFS benefit over trastuzumab when combined with chemotherapy, no comparisons exist between this combo and the novel, recently approved anti-HER2 regimens.

In this regard, the tucatinib triplet demonstrated to improve OS over standard-of-care [25], while trastuzumab deruxtecan showed unprecedented activity in highly pretreated patients [5]; both regimens, thus, currently represent more appealing choices in patients pretreated with TDM-1, and are recommended as treatment options by the latest guidelines[4]. More in detail, the ORR (60.9\%), duration of response (14.8 months) and PFS (16.4 months) demonstrated by trastuzumab deruxtecan appear as the highest ever achieved in this setting [5]; although these results were obtained in a non-randomized trial, various randomized phase 3 trials are ongoing to confirm the finding[3]. Tucatinib instead was formally compared to standard-of-care in a well-designed randomized trial, demonstrating to double ORR ( $40.6 \%$ vs $22.8 \%$ ), improve OS by 4.5 months ( 21.9 vs 17.4 months) as well as achieve a very relevant activity in patients with treated and untreated brain metastasis $[6,26]$.

Nonetheless, both these regimens also harbor significant toxicities, including gastrointestinal toxicity and fatigue for both, palmar-plantar erythrodysesthesia for tucatinib triplet and alopecia for trastuzumab deruxtecan. The same applies to the recently approved neratinib, which showed relatively high rates of diarrhea, nausea and vomiting in the NALA trial[7]. Conversely, margetuximab showed an optimal safety profile, comparable with that of trastuzumab, a factor that may influence its future impact on treatment algorithms[22].

Based on abovementioned considerations, a role for margetuximab may be hypothesized in the future for advanced HER2positive $B C$ patients progressing following standard firstand second-line regimens, as well as tucatinib and/or trastuzumab deruxtecan, although current guidelines do not yet recommend treatment with margetuximab in any setting[4]. Notably, the differential activity of the compound based on CD16A allele may lead to its preference in the subpopulation carrying the CD16A-158 F allele (about 85\% of the patients) [22], despite the determination of CD16A allele could likely add non-negligible time and costs to the management of patients, possibly representing a barrier to the implementation of the compound in lowand middle-income countries. Similar considerations regarding financial toxicity applies to the potential costs of the drug compared with trastuzumab. Indeed, the price of newly approved cancer drugs is progressively increasing, with no apparent relationship with the efficacy demonstrated in clinical trials[27]. In this perspective, with the availability of relatively affordable biosimilars of trastuzumab, the modest improvement in PFS demonstrated in the SOPHIA trial may not justify the difference in price required for the use of margetuximab, particularly in those countries with a public-based healthcare system.

Ultimately, a better picture of the clinical role of margetuximab will require updated efficacy data, to determine if the compound is able to improve OS or if its benefit is restricted to a modest prolongation of PFS. In the latter case, less costly combinations of chemotherapy and trastuzumab may be preferred after pretreatment with the standard-of-care. 
Regardless of the clinical future of the compound, its developmental pathway has produced significant advancements on our knowledge of $\mathrm{BC}$. Indeed, the prospective demonstration of a differential activity of margetuximab based on CD16A allele has both highlighted the implication of patient's genotype in determining treatment outcomes, as well as the relevance of ADCC in the context of HER2-blockade. Although previously hypothesized [14], SOPHIA trial is the first prospective trial demonstrating the importance of CD16A genotype as a predictor of efficacy to anti-HER2 treatments, a paradigm for which further explorations are warranted, with the aim of better tailoring treatments based on patients' characteristics. The role of ADCC could instead progressively become more and more relevant with the emergence of immunotherapy combinations with anti-HER2 agents, some of which have already shown encouraging clinical results.

\section{Conclusion}

The PFS improvement demonstrated in the SOPHIA trial has launched margetuximab on the crowded field of novel antiHER2 agents demonstrating to improve outcomes in pretreated HER2-positive advanced BC. Updated efficacy data will be needed to clarify the impact of margetuximab on OS, as well as to indirectly compare its activity with other anti-HER2 agents which currently appear as the optimal treatment choices in TDM1-pretreated patients, including tucatinib and trastuzumab deruxtecan. Regardless of the clinical implementation of margetuximab, the improved biological understanding derived from its development is expected to fuel new paradigms in BC treatment, with the purpose of optimally tailoring treatments based on patients' characteristics and exploiting the immune-mediated effects of anti-HER2 compounds.

\section{Drug Summary Box}

Drug name: Margetuximab

Phase: III

Indication: pretreated HER2-positive advanced breast cancer

Mechacnism of action: HER2 pathway disruption; antibodydependent cellular cytotoxicity activation, through an engineered FC domain improving binding to the activating receptor FcyRIIIA

Chemical structure: HER2-targeting monoclonal antibody

Pivotal trials: phase 3 "SOPHIA" trial [21]

\section{Funding}

This paper is not funded.

\section{Declaration of interest}

G Curigliano received honoraria for speaker, consultancy or advisory roles from Roche, Pfizer, Novartis, Seattle Genetics, Lilly, Ellipses Pharma, Foundation Medicine and Samsung. The authors have no other relevant affiliations or financial involvement with any organization or entity with a financial interest in or financial conflict with the subject matter or materials discussed in the manuscript apart from those disclosed.

\section{Reviewer disclosures}

Peer reviewers on this manuscript have no relevant financial relationships or otherwise to disclose.

\section{References}

1. Owens MA, Horten BC, Da Silva MM. HER2 amplification ratios by fluorescence in situ hybridization and correlation with immunohistochemistry in a cohort of 6556 breast cancer tissues. Clin Breast Cancer. 2004;5(1):63-69.

2. Pernas S, Tolaney SM. HER2-positive breast cancer: new therapeutic frontiers and overcoming resistance. Ther Adv Med Oncol. 2019;11:175883591983351.

3. Cesca MG, Vian L, Cristóvão-Ferreira $S$, et al. HER2-positive advanced breast cancer treatment in 2020. Cancer Treat Rev. 2020;88:102033.

4. Cardoso F, Paluch-Shimon S, Senkus E, et al. 5th ESO-ESMO international consensus guidelines for advanced breast cancer (ABC 5) $\dagger$. Annals of Oncology. 2020;31(12):1623-1649.

5. Modi S, Saura C, Yamashita T. et al. Trastuzumab Deruxtecan in Previously Treated HER2-Positive Breast Cancer. N Engl J Med. 2019. DOI:10.1056/NEJMoa1914510.

6. Murthy RK, Loi S, Okines A et al. Tucatinib, Trastuzumab, and Capecitabine for HER2-Positive Metastatic Breast Cancer. N Engl J Med. 2019;NEJMoa1914609. DOI:10.1056/NEJMoa1914609.

7. Saura C, Oliveira M, Feng YH et al. Neratinib Plus Capecitabine Versus Lapatinib Plus Capecitabine in HER2-Positive Metastatic Breast Cancer Previously Treated With $\geq 2$ HER2-Directed Regimens: phase III NALA Trial. J Clin Oncol. 2020;147. DOI:10.1200/JCO.20.00147.

8. Cardoso F, Senkus E, Costa A et al. 4th ESO-ESMO International Consensus Guidelines for Advanced Breast Cancer (ABC 4). Ann. Oncol. 2018;29(8):1634-1657.

9. Lin NU, Borges V, Anders C et al. Intracranial Efficacy and Survival With Tucatinib Plus Trastuzumab and Capecitabine for Previously Treated HER2-Positive Breast Cancer With Brain Metastases in the HER2CLIMB Trial. J Clin Oncol. 2020;00775. DOI:10.1200/ JCO.20.00775.

10. Emens LA, Esteva FJ, Beresford $M$, et al. 3050Overall survival (OS) in KATE2, a phase II study of programmed death ligand 1 (PD-L1) inhibitor atezolizumab (atezo)+trastuzumab emtansine (T-DM1) vs placebo (pbo)+T-DM1 in previously treated HER2+ advanced breast cancer (BC). Ann Oncol. 2019;30:v104.

11. Stavenhagen JB, Gorlatov S, Tuaillon N, et al. Fc Optimization of Therapeutic Antibodies Enhances Their Ability to Kill Tumor Cells In vitro and Controls Tumor Expansion In vivo via Low-Affinity Activating Fc Receptors. Cancer Res. 2007;67(18):8882-8890.

12. Nordstrom JL, Gorlatov S, Zhang W, et al. Anti-tumor activity and toxicokinetics analysis of MGAH22, an anti-HER2 monoclonal antibody with enhanced Fcy receptor binding properties. Breast Cancer Res. 2011;13(6):R123.

13. Lehrnbecher T, Foster CB, Zhu S, et al. Variant Genotypes of the Low-Affinity Fcy Receptors in Two Control Populations and a Review of Low-Affinity Fcy Receptor Polymorphisms in Control and Disease Populations. Blood. 1999;94(12):4220-4232.

14. Musolino A, Naldi N, Bortesi B, et al. Immunoglobulin G Fragment C Receptor Polymorphisms and Clinical Efficacy of TrastuzumabBased Therapy in Patients With HER-2/neu -Positive Metastatic Breast Cancer. J. Clin. Oncol. 2008;26(11):1789-1796.

15. Pereira NA, Chan KF, Lin PC, et al. The "less-is-more" in therapeutic antibodies: afucosylated anti-cancer antibodies with enhanced antibody-dependent cellular cytotoxicity. MAbs. 2018;10:693-711.

16. Tarantino P, Hamilton E, Tolaney SM, et al. HER2-Low Breast Cancer: pathological and Clinical Landscape. J Clin Oncol. 2020;38 (17):1951-1962.
Q6

Q7

Q8

Q9

Q10

Q11

Q12

Q13 
17. Bang YJ, Giaccone G, Im SA, et al. First-in-human phase 1 study of margetuximab (MGAH22), an Fc-modified chimeric monoclonal antibody, in patients with HER2-positive advanced solid tumors. Ann. Oncol. 2017;28(4):855-861.. Note: First in human trial of Margetuximab

18. Leveque D, Gigou L, Bergerat J. Clinical Pharmacology of Trastuzumab. Curr Clin Pharmacol. 2008;3:51-55.

19. https://clinicaltrials.gov/ct2/show/results/NCT01828021 Accessed on 23 Sept 2020.

20. Catenacci DVT, Kang Y-K, Park H, et al. Margetuximab plus pembrolizumab in patients with previously treated, HER2-positive gastro-oesophageal adenocarcinoma (CP-MGAH22-05): a single-arm, phase 1b-2 trial. Lancet Oncol. 2020;21(8):1066-1076.

21. Rugo HS, Im S-A, Wright GLS, et al. SOPHIA primary analysis: A phase 3 (P3) study of margetuximab (M) + chemotherapy (C) versus trastuzumab $(T)+C$ in patients (pts) with HER2+ metastatic (met) breast cancer (MBC) after prior anti-HER2 therapies (TX). J. Clin. Oncol. 2019;37(15_suppl):1000..• First analysis of phase 3 SOPHIA trial, comparing Margetuximab to standard-of-care

22. Rugo HS, Im SA, Cardoso F et al. Abstract GS1-02: phase 3 SOPHIA study of margetuximab + chemotherapy vs trastuzumab + chemotherapy in patients with HER2+ metastatic breast cancer after prior anti-HER2 therapies: second interim overall survival analysis. General Session Abstracts GS1-02-GS1-02 (American Association for Cancer Research, 2020) 2020. doi:10.1158/1538-7445.SABCS19-GS1 $-02$.

23. Escrivá S, Im S-A, Cardoso F, et al. SOPHIA analysis by chemotherapy (Ctx) choice: A phase III (P3) study of margetuximab (M) + Ctx versus trastuzumab $(T)+C t x$ in patients (pts) with pretreated HER2 + metastatic (met) breast cancer (MBC). J. Clin. Oncol. 2020;38 (15_suppl): 1040.

24. Tarantino P, Morganti S, Curigliano G. Biologic therapy for advanced breast cancer: recent advances and future directions. Expert Opin Biol Ther. 2020;20:1009-1024.
25. Murthy R, Borges VF, Conlin A, et al. Tucatinib with capecitabine and trastuzumab in advanced HER2-positive metastatic breast cancer with and without brain metastases: a non-randomised, open-label, phase 1b study. Lancet Oncol. 2018;19(7):880-888.

26. Lin NU, Borges V, Anders C et al. Intracranial Efficacy and Survival With Tucatinib Plus Trastuzumab and Capecitabine for Previously Treated HER2-Positive Breast Cancer With Brain Metastases in the HER2CLIMB Trial. J Clin Oncol. 2020;775. DOI:10.1200/JCO.20.00775.

27. Mailankody S, Prasad V. Five Years of Cancer Drug Approvals. JAMA Oncol. 2015;1:539.

28. Verma S, Miles D, Gianni L, et al. Trastuzumab Emtansine for HER2-Positive Advanced Breast Cancer. N. Engl. J. Med. 2012;367 (19):1783-1791.

29. Blackwell KL, Burstein HJ, Storniolo AM, et al. Randomized Study of Lapatinib Alone or in Combination With Trastuzumab in Women With ErbB2-Positive, Trastuzumab-Refractory Metastatic Breast Cancer. J. Clin. Oncol. 2010;28(7):1124-1130.

30. Pivot X, Manikhas A, Żurawski B, et al. CEREBEL (EGF111438): A Phase III, Randomized, Open-Label Study of Lapatinib Plus Capecitabine Versus Trastuzumab Plus Capecitabine in Patients With Human Epidermal Growth Factor Receptor 2-Positive Metastatic Breast Cancer. J. Clin. Oncol. 2015;33(14):1564-1573.

31. Tolaney SM, Wardley AM, Zambelli $S$, et al. A monarcHER: a Randomized Phase 2 study of Abemaciclib plus Trastuzumab with or without fulvestrant versus trastuzumab plus standard of care chemotherapy in women with HR+, HER2+ Advanced Breast Cancer (ABC). Ann Oncol. 2019;30:v851-v934.

32. Park $\mathrm{YH}$, Lee $\mathrm{K}-\mathrm{H}$, Sohn JH, et al. A phase II trial of the pan-HER inhibitor poziotinib, in patients with HER2-positive metastatic breast cancer who had received at least two prior HER2-directed regimens: results of the NOV120101-203 trial. Int J Cancer. 2018;143(12):3240-3247.

33. Xu B, Yan M, Ma F, et al. Pyrotinib or lapatinib plus capecitabine for HER2+ metastatic breast cancer (PHOEBE): A randomized phase III trial. J. Clin. Oncol. 2020;38(15_suppl):1003. 